\title{
The Hyperbolic and Linear Phases of the Sucrose Accumulation Curve in Turnip Storage Cells Denote Carrier-mediated and Fluid Phase Endocytic Transport, Respectively
}

\author{
Diego Pozueta-Romero, Pedro Gonzalez, and Ed Etxeberria ${ }^{\mathbf{1}}$ \\ Horticulture Sciences Department, University of Florida, IFAS, Citrus Research \\ and Education Center, Lake Alfred, FL 33850

\begin{abstract}
Javier Pozueta-Romero
Instituto de Agrobiotecnología, Consejo Superior de Investigaciones Científicas/Universidad

Pública de Navarra/Gobierno de Navarra, Mutiloako etorbidea zembaki gabe, 31192 Mutiloabeti, Nafarroa, Spain
\end{abstract}

\begin{abstract}
AdDitional INDEX words. Brassica campestris, basal endocytosis, sucrose transporter, sucrose uptake
Abstract. Sucrose accumulation into sink cells consists of multiple components collectively characterized by a biphasic kinetics curve. Whereas the hyperbolic phase at low external sucrose concentration denotes a membranebound, carrier-mediated component, the linear nonsaturable phase at higher concentrations has been suggested to represent facilitated diffusion. We recently demonstrated the occurrence of fluid phase endocytosis (FPE) uptake of sucrose in heterotrophic cells. To investigate the involvement of this process within both phases of the sucrose accumulation curve, we analyzed the effect of phloridzin and latrunculin-B (sucrose $/ \mathrm{H}^{+}$symport and endocytosis inhibitors, respectively) in the accumulation of sucrose and the endocytic marker Alexa-488 in turnip (Brassica campestris L.) storage parenchyma cells. At low external sucrose concentration, phloridzin, but not latrunculin-B, greatly reduced sucrose accumulation. By contrast, at high external sucrose concentration, phloridzin and latrunculin-B significantly inhibited sucrose accumulation. In addition, latrunculin-B exerted a pronounced inhibitory effect on Alexa-488 uptake at any external sucrose concentration. Our results indicate that carrier-mediated and endocytic uptake (at different magnitudes) of sucrose take place at any external sucrose concentration; that within the hyperbolic phase of the sucrose accumulation curve, most of the sucrose accumulating in the cell enters via plasmalemmabound carrier(s); and that within the linear phase, plasmalemma-bound carriers and FPE are determinants of sucrose accumulation, with the involvement of FPE increasing parallel to external sucrose. Confocal laser scanning microscopy observations confirmed the increased involvement of FPE at higher external sucrose concentrations.
\end{abstract}

In higher plants, sucrose constitutes the main form of photoassimilate transport from source leaves to sink tissues (Lalonde et al., 1999). Biochemical studies have demonstrated that sucrose accumulation into sink cells consists of multiple components collectively characterized by a biphasic kinetics curve (Bieleski, 1960; Chapleo and Hall, 1989; Daie, 1984; Saftner and Daie, 1983; Wright and Oparka, 1989). The two continuous but distinctive phases denote the involvement of two independent transport systems. Whereas the hyperbolic phase at low external sugar concentration is believed to represent a high-affinity, membrane-bound, carrier-mediated component, the linear nonsaturable phase at higher concentrations has been suggested to represent facilitated diffusion (Daie, 1986; Niemietz and Jenner, 1993; Wright and Oparka, 1989), presumably mediated by a sucrose-binding protein (Overvoorde et al., 1996).

Sucrose transporters have been identified and thoroughly examined as part the phloem-loading mechanism (Lalonde et al., 2004), with fewer reports demonstrating their existence at the plasmalemma (Manning, 1998; Manning et al., 2001; Shakya and Sturm, 1998) and tonoplast of storage cells (Endler et al., 2006; Getz and Klein, 1995; Shimoaka et al., 2004). Their

Received for publication 21 Dec. 2007. Accepted for publication 27 Mar. 2008. ${ }^{1}$ Corresponding author. E-mail: eje@crec.ifas.ufl.edu presence in both membrane systems, and in tissue uptake kinetics, strongly support their role in the process of sucrose uptake and accumulation. However, a growing volume of evidence argues that metabolite transport from the apoplast cannot be fully reconciled by solute movement through channels or transporters, but in some instances, the data imply the presence of alternate or parallel transport mechanisms (Fernie et al., 2000; Haug and Shi, 1991; MacRobbie, 1999; Wyse, 1979).

A vesicle-mediated fluid phase endocytic (FPE) system for solute transport has been described for citrus (Citrus L.) juice cells (Etxeberria et al., 2005b), sycamore (Acer pseudoplatanus L.) cultured cells (Etxeberria et al., 2005a; 2006), and celery (Apium graveolens L.) petiole storage parenchyma (Etxeberria et al., 2007). Aside from its bulk transport capacity, a distinguishing characteristic of FPE is its linear, nonsaturable transport kinetics (Basrai et al., 1990). Some details of FPE working in parallel with plasmalemma carriers to transport sugars to different cellular compartments were described using cultured cells of sycamore (Etxeberria et al., 2005a, 2005c). The presence of these two systems for sugar transport into storage cells, and the observation that FPE in celery parenchyma was only induced at high external mannitol concentrations (Etxeberria et al., 2007a), prompted us to re-examine the possible role of FPE and membrane-carrier transport within 
both phases of the characteristic accumulation curve. To study the interrelationship between sucrose uptake mechanisms, we selected turnip hypocotyls, not only for having sucrose as a main form of carbon transport (Nishikawa et al., 2005), but for the lack of storage starch, which could potentially complicate measurements of sucrose uptake.

In this article, we present evidence indicating that the hyperbolic phase of sucrose accumulation denotes carriermediated transport, whereas the linear phase represents a superimposed system with a significant FPE component.

\section{Materials and Methods}

\section{Plant material}

Turnip hypocotyls were purchased at a local grocery store and were kept refrigerated at $40{ }^{\circ} \mathrm{C}$ until use. Hypocotyls were cut in half and tissue cylinders were obtained using a cork borer ( $3 \mathrm{~mm}$ in diameter). The cylinders were sliced into $0.5-\mathrm{mm}$ thick discs and were rinsed with a solution containing $25 \mathrm{~mm}$ Tris/MES (pH 5.6), $0.5 \mathrm{~mm} \mathrm{CaCl}_{2}, 0.5 \mathrm{~mm} \mathrm{MgCl}_{2}$, and $250 \mathrm{~mm}$ betaine (starving media). The tissue was blot dried and weighed accordingly.

Determination of intracellular Sucrose CONTENT. Tissue samples $(3 \mathrm{~g}$ ) were preincubated for $30 \mathrm{~min}$ in 10 - $\mathrm{mL}$ flasks with $3 \mathrm{~mL}$ of starving medium at $21^{\circ} \mathrm{C}$ and $80 \mathrm{rpm}$. After a 30 min preincubation, the starving solution was replaced by fresh incubation medium containing sucrose, and the tissue was incubated for an additional $5 \mathrm{~h}$. The combined concentration of sucrose plus betaine always remained at $250 \mathrm{~mm}$. After $5 \mathrm{~h}$, the incubation medium was discarded and the tissue was washed three times (10 min per wash) with $5 \mathrm{~mL}$ of starving medium. The final wash solution consisted of $5 \mathrm{~mL}$ of $80 \%$ ethanol, and the tissue was sonicated for $15 \mathrm{~s}$ to stop all metabolic activity. When indicated, inhibitors were also added to the preincubation and incubation medium. Latrunculin-B at $10 \mu \mathrm{m}$ and phloridzin at $2 \mathrm{~mm}$ were used as endocytic (Baluska et al., 2002) and sucrose $/ \mathrm{H}^{+}$symport inhibitors (Bush, 1993), respectively. Sucrose was determined using the modified anthrone method described by Van Handel (1968).

UPTAKE OF ALEXA-488. Experiments to determine the uptake of the endocytic marker Alexa-488 (Emans et al., 2002) were carried out essentially as described above for sucrose, except that the incubation medium also contained $100 \mu \mathrm{M}$ Alexa-488 (Molecular Probes, Eugene, OR). After a 5-h incubation, protoplasts were prepared as described below and were fluorescence analyzed using a Versafluor fluorometer (Bio-Rad Laboratories, Hercules, CA) with an excitation filter of 490/10 and emission filter of 510/10. Conversion of fluorescence to units of relative fluorescence was based on a predetermined standard curve. Alexa-488 values are presented based on the cytosolic marker alcohol dehydrogenase (ADH) as a means to standardize the database on the amount of live cells. ADH activity was measured at $340 \mathrm{~nm}$ essentially as described by Etxeberria et al. (2005a). One unit (U) is the amount of activity that consumes $1 \mu \mathrm{mol}$ ethanol per minute.

Protoplast preparation. Protoplasts were obtained by incubating the tissue in starving medium supplemented with 800 mm mannitol, 2\% cellulase (Serva Electrophoresis, Heidelberg, Germany), 1\% macerase (Serva Electrophoresis), and $0.5 \%$ pectinase (Fluka Biochemika, Buchs, Switzerland). After $4 \mathrm{~h}$ of enzymatic digestion, protoplasts were washed three times with $2.5 \mathrm{~mL}$ of a similar solution without enzymes, resuspended in $2 \mathrm{~mL}$ of this solution, and sonicated for $15 \mathrm{~s}$. The resulting suspension was centrifuged at $13,000 g_{\mathrm{n}}$ for $5 \mathrm{~min}$ and the supernatant was collected for analysis. All experiments were carried out in triplicates, and data is presented as the average \pm SD.

CONFOCAL LASER SCANNING MicrosCopy. A final suspension of protoplasts, prepared as described above, was resuspended in a 3-mL solution of $25 \mathrm{~mm}$ MES (pH 5.6), $1 \mathrm{~mm} \mathrm{CaCl}_{2}, 700 \mathrm{M}$ betaine, $100 \mathrm{~mm}$ sucrose, $100 \mu \mathrm{M}$ Alexa 488, and $15 \mu_{\mathrm{M}}$ FM4 64 (Molecular Probes), and was incubated at $21{ }^{\circ} \mathrm{C}$ for different periods of times. Protoplasts were observed with a TCS-SL confocal-fluorescent scanning microscope (Leica, Heidelberg, Germany).

\section{Results}

Sucrose accumulation. Sucrose accumulation in turnip storage parenchyma cells incubated at increasing external concentrations resulted in the traditional biphasic curve (Fig. 1; Bieleski, 1960; Saftner and Daie, 1983). An unmistakable hyperbolic phase of rapid sucrose accumulation leveled off at around $10 \mathrm{~mm}$, with a linear phase commencing at $\approx 40 \mathrm{~mm}$. This concentration, at which the linear phase presumptively begins, was calculated as the inflection point between an extended hyperbolic curve generated using concentrations up to $50 \mathrm{~mm}$, and a linear phase from 50 to $300 \mathrm{~mm}$ (Fig. 1). Sucrose content at 10 to $40 \mathrm{~mm}$ external sucrose was $\approx 700 \mu \mathrm{g}$ of sucrose per $1 \mathrm{~g}$ of fresh weight (FW), and increasing the concentration to $300 \mathrm{~mm}$ only resulted in an additional increment of $\approx 600 \mu \mathrm{g}$ of sucrose per $1 \mathrm{~g}$ of FW (Fig. 1).

EFFECT OF INHIBITORS ON SUCROSE ACCUMULATION. To examine the effect of endocytic and membrane-bound carrier inhibitors on each phase of sucrose accumulation, two sucrose concentrations were selected. These concentrations (5 and 100 $\mathrm{mm}$ for the hyperbolic and linear phase, respectively) were selected to represent a distinctive point of each phase. The actin-depolymerizing agent latrunculin-B was used as an

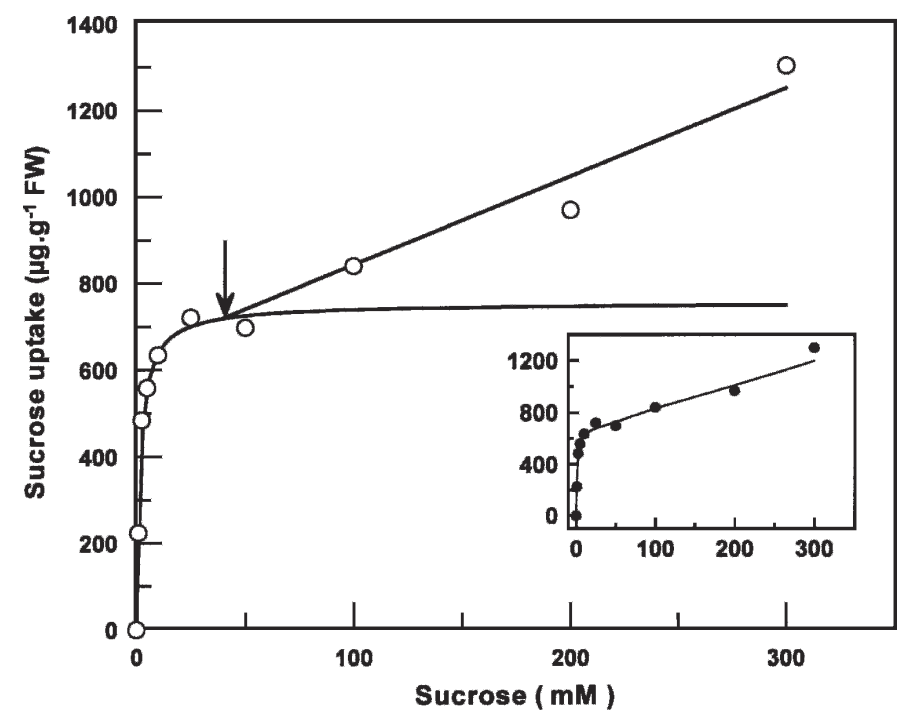

Fig. 1. Combined hyperbolic and linear phases of sucrose accumulation into discs of turnip storage parenchyma cells. The extended hyperbolic curve was calculated using concentrations up to $50 \mathrm{~mm}$, and the linear phase was calculated using concentrations from 50 to $300 \mathrm{~mm}$. Combined, all points generate a biphasic curve (inset). 
endocytic inhibitor (Baluska et al., 2004), whereas phloridzin was used as a sucrose $/ \mathrm{H}^{+}$symport inhibitor, as demonstrated by Bush (1993).

Sucrose accumulation at $5 \mathrm{~mm}$ external concentration was completely abolished by 2 mм phloridzin, whereas latrunculin$\mathrm{B}$ had little effect under similar conditions (Fig. 2). When the concentration of sucrose was increased to $100 \mathrm{~mm}$, sucrose content was reduced $53 \%$ by phloridzin and $23 \%$ by latruncuinB. It is worth noting that at the latter concentration, most of the sucrose accumulation is determined by the hyperbolic phase, as indicated in Fig. 1.

EFFECT OF INHIBITORS ON FPE MARKER ACCUMULATION. TO verify the involvement of FPE within both phases of sucrose accumulation, we measured the uptake of the soluble endocytic marker Alexa-488 (Emans et al., 2002) by turnip storage cells at 5 and $100 \mathrm{~mm}$ external sucrose. Because Alexa-488 binds to cell walls, potentially introducing artifacts during the extraction of internalized Alexa-488, we generated protoplasts after tissue incubation. At $5 \mathrm{~mm}$ sucrose, latrunculin-B, but not phloridzin, exerted a strong inhibitory effect on Alexa-488 accumulation (Fig. 3). The low levels of Alexa-488 accumulated at this concentration (and strongly inhibited by latrunculin-B) implicate the participation of a basal level endocytic activity under these conditions. Similar results were obtained with sycamore cultured cells and celery petiole parenchyma incubated in the absence of sucrose and mannitol, respectively (Etxeberria et al., 2005a, 2007a). A basal level of endocytosis in plant cells is believed to be responsible for the continuous turnover of membrane components (Robinson et al., 1998), the retrieval and desensitization of receptors for signal dispersal (Piddini and Vincent, 2003; Sorkin and von Zastrow, 2002), and the

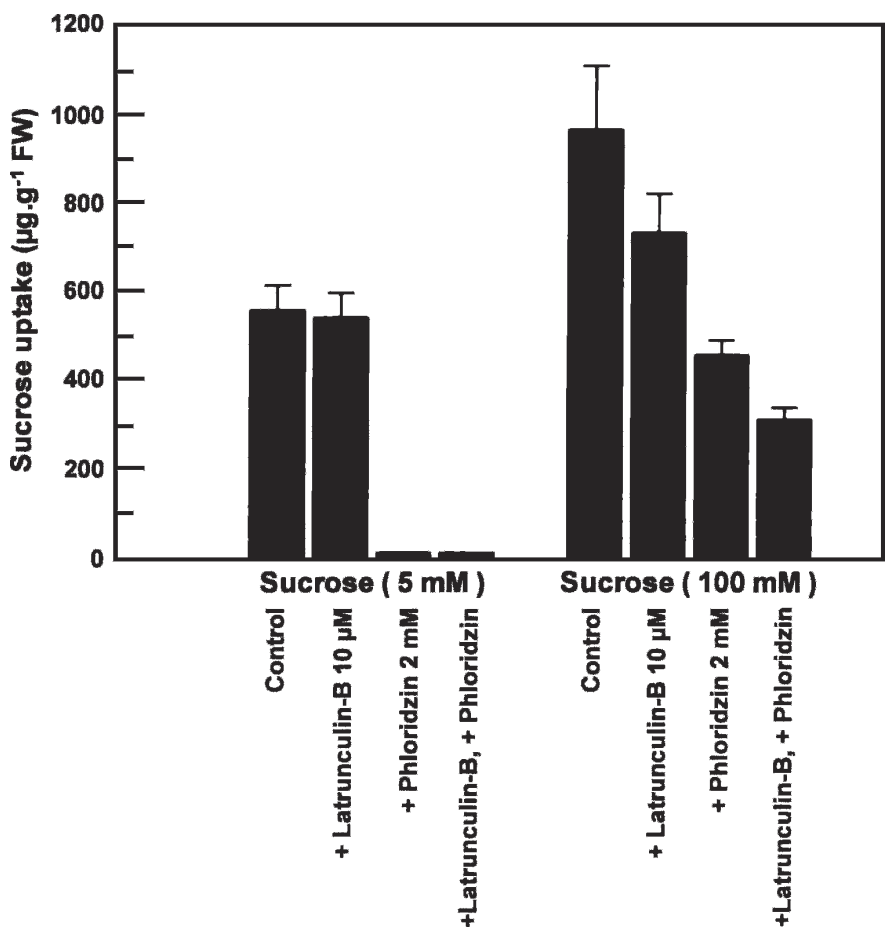

Fig. 2. Sucrose accumulation turnip storage parenchyma cells at 5 and $100 \mathrm{~mm}$ external sucrose concentrations in the absence and presence of $2 \mathrm{~mm}$ phloridzin or $10 \mu \mathrm{M}$ latrunculin-B. Tissue was preincubated for $30 \mathrm{~min}$ with or without the inhibitors before the addition of sucrose.

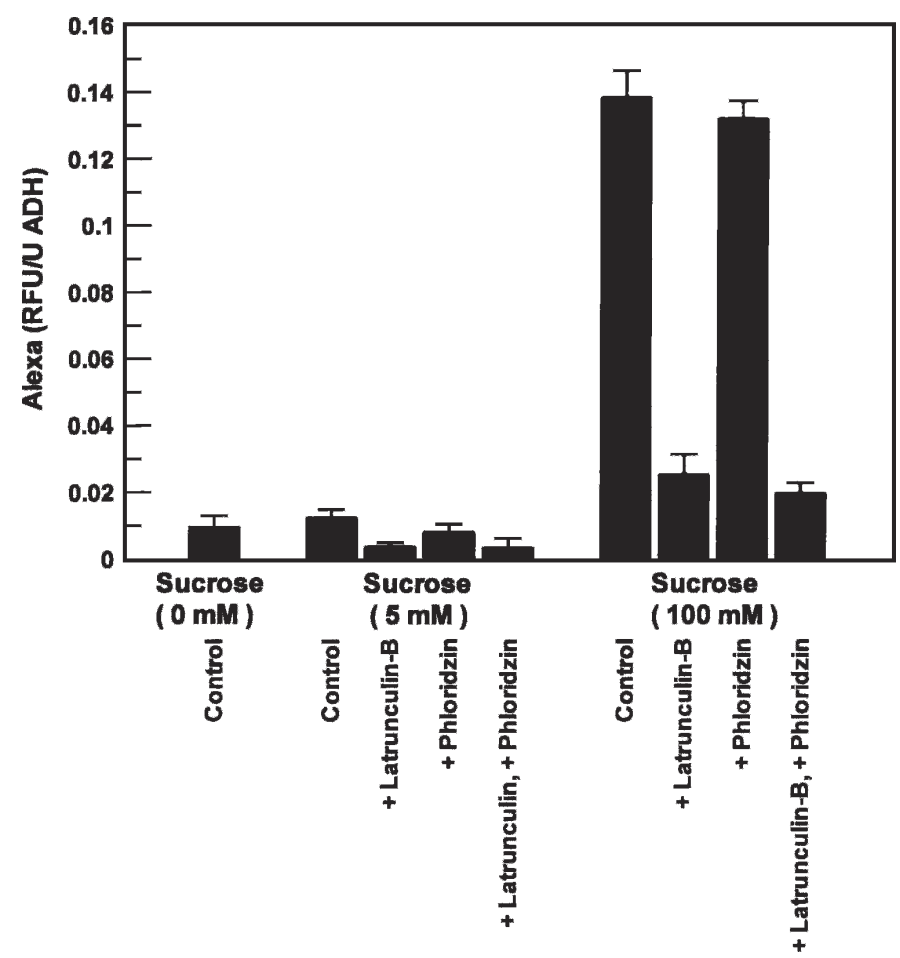

Fig. 3. Accumulation of Alexa-488 by turnip storage parenchyma cells at 0,5 , and $100 \mathrm{~mm}$ external sucrose concentrations in the absence and presence of $2 \mathrm{~mm}$ phloridzin or $10 \mu \mathrm{M}$ latrunculin-B. Protoplasts were prepared before Alexa-488 extraction, determination, and ADH measurements as described in "Materials and Methods."

dynamic redistribution of cell wall pectins (Baluska et al., 2002).

The uptake of Alexa-488 increased 10-fold over control (no sucrose) and $5 \mathrm{~mm}$ sucrose when external sucrose was increased to $100 \mathrm{~mm}$. At this concentration, the uptake of Alexa-488 was reduced $\approx 85 \%$ by latrunculin-B, whereas phloridzin had no inhibitory effect (Fig. 3). These results are consistent with an increased endocytic transport activity at higher external sucrose concentrations.

The participation of FPE in the accumulation of sucrose at high external concentrations was verified by confocal fluorescent scanning microscopy observations of cells incubated in $100 \mathrm{~mm}$ sucrose, the endocytic marker Alexa-488, and the membrane marker FM 4-64. FM 4-64 is a membrane-impermeable polar fluorochrome that fluoresces upon intercalation to the outer leaflet of the plasma membrane. Internalization of the fluorescent plasmalemma serves as tracer for endocytosis and of vesicle trafficking (Fischer-Parton et al., 2000). To avoid interfering with the background, protoplasts were washed with unlabeled incubating solution before observation. In Fig. 4, two types of endocytic structures are evident, the smaller vesicles of $\approx 1$ to $2 \mu \mathrm{m}$ likely representing endocytic vesicles (Etxeberria et al., 2007b). The larger $\approx 9-\mu \mathrm{m}$ organelles are more difficult to identify given their lack of internal structural clarity, but appear to be intermediate structures in the endocytic process. In all labeled structures, the red fluorescent membranes are indicative of their plasmalemma origin, and the fluorescent contents demonstrate the external nature of the fluids.

A later stage of the endocytic process is presented in Fig. 5. After the endocytic process culminates with the final deposition 

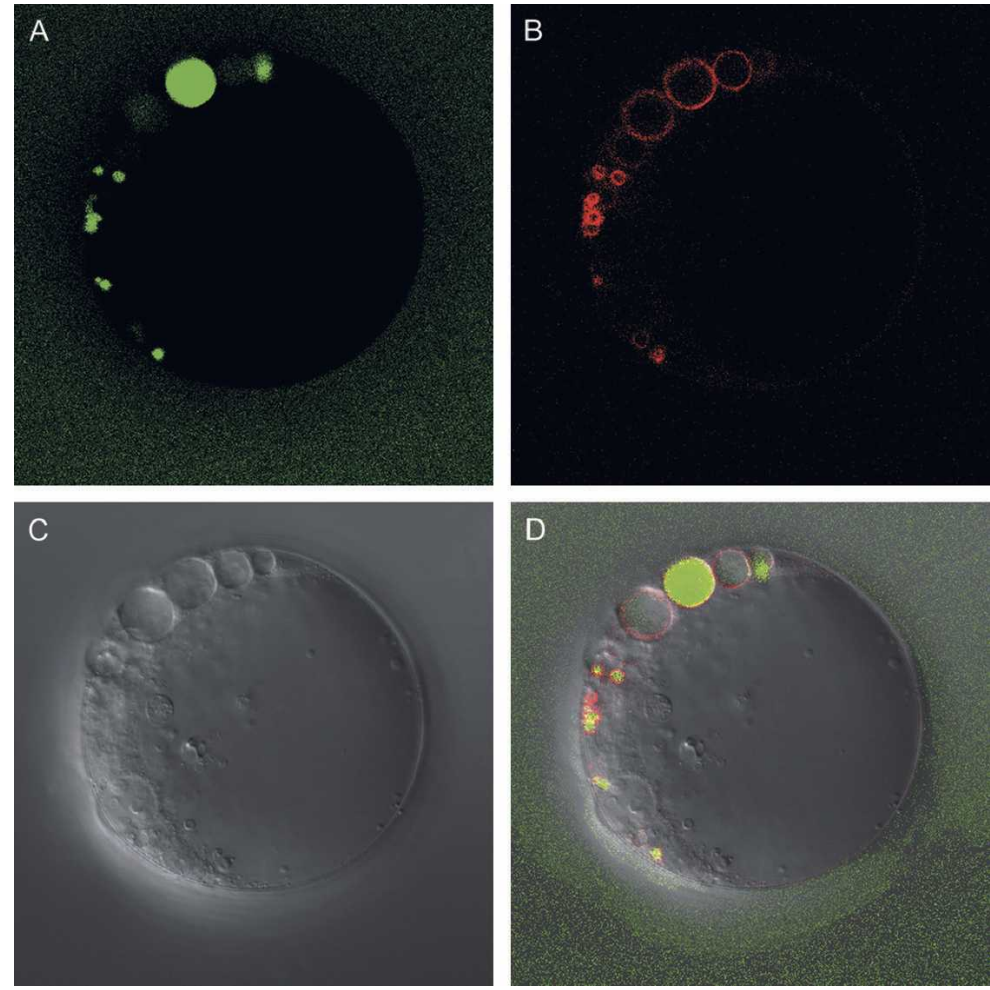

Fig. 4. High-definition confocal microscopy of a turnip protoplast incubated for $4 \mathrm{~h}$ in a solution containing $100 \mathrm{~mm}$ sucrose, $100 \mu \mathrm{M}$ Alexa-488, and $15 \mu \mathrm{M}$ FM4-64. Protoplasts were washed to eliminate background interference. Confocal microscope was set to detect the fluorescence emission of both dyes at (A) $488 \mathrm{~nm}$ for Alexa-488 and (B) $750 \mathrm{~nm}$ for FM 4-64. (C) represents a white light image. (D) is a superimposed composite image of A through $\mathrm{C}$.
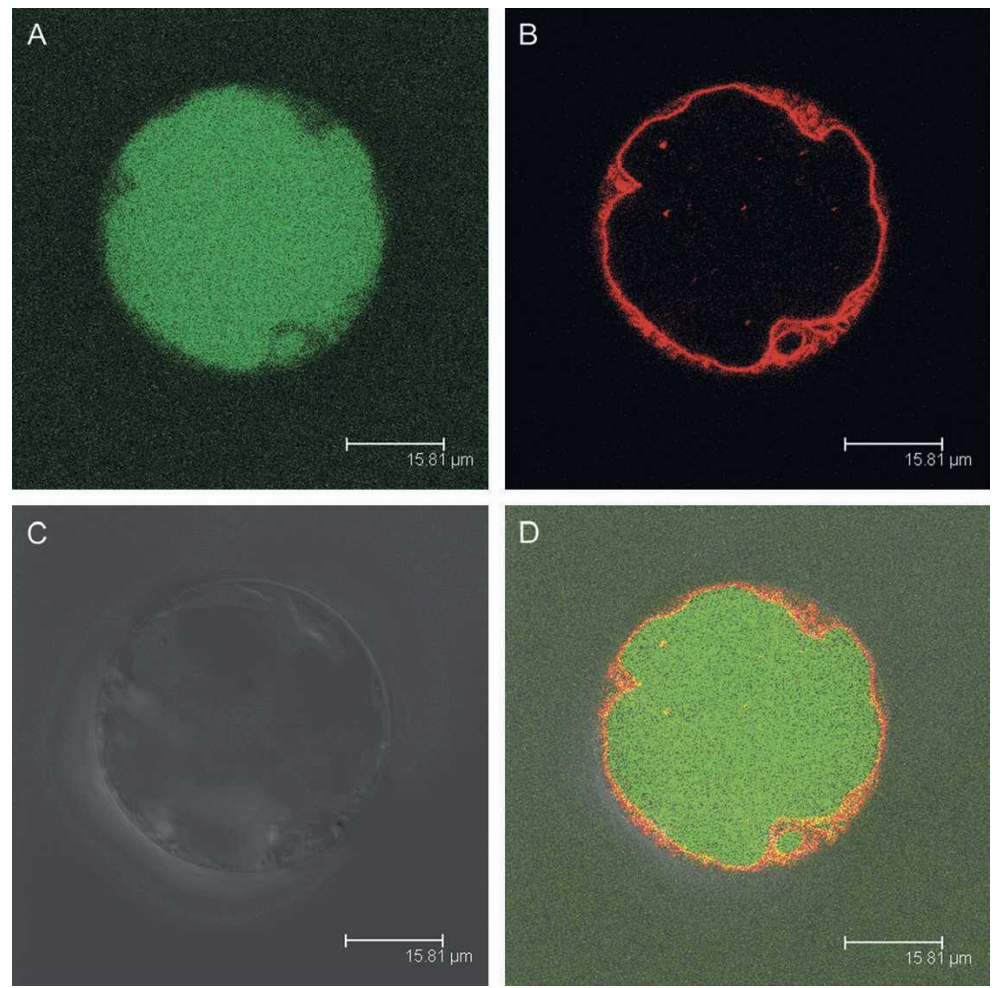

Fig. 5. High-definition confocal microscopy of a turnip protoplast incubated for $12 \mathrm{~h}$ in a solution containing $100 \mathrm{~mm}$ sucrose, $100 \mu \mathrm{M}$ Alexa-488, and $15 \mu \mathrm{M}$ FM4-64. Protoplasts were washed to eliminate background interference. Confocal microscope was set to detect the fluorescence emission of both dyes at (A) $488 \mathrm{~nm}$ for Alexa-488 and (B) $750 \mathrm{~nm}$ for FM 4-64. (C) represents a white light image. (D) is a superimposed composite image of A through C. of sucrose and Alexa-488 in the vacuole, its contents and the tonoplast are highly labeled. At this point, other membranous structures become labeled, given the amplitude of the membrane recycling process. The presence of latrunculin-B prevented internalization of the plasmalemma and transport of external solutes into the vacuole.

F P E - I N D E P E N D E N T S U C R O S E acCumulation. The ubiquitous presence of basal endocytic transport even in the absence of external sucrose (as part of membrane recycling) prevents us from unequivocally allocating membrane-bound transporters an exclusive role in sucrose accumulation at low external sucrose concentration. To determine the percentage of sucrose transported by basal endocytosis at lower sucrose concentrations (below $10 \mathrm{~mm}$; see Fig. 1), we incubated turnip cells in the presence of $1 \mathrm{~mm}$ sucrose and latrunculin-B. At this low sucrose concentration, FPE involvement is excluded, and the presence of latrunculin-B would block any basal endocytic transport into the vacuole (Fig. 2; Etxeberria et al., 2007a). The difference in sucrose accumulation between tissues incubated in the absence and in the presence of latrunculin-B shown in Fig. 6 represents total sucrose transported and accumulated by basal endocytosis. At $6 \mathrm{~h}$ of incubation, for example, tissue accumulated $\approx 190 \mu \mathrm{g}$ of sucrose per $1 \mathrm{~g}$ of FW. In the presence of latrunculin$\mathrm{B}$, incubation was reduced to $135 \mu \mathrm{g}$ of sucrose per $1 \mathrm{~g}$ of $\mathrm{FW}$. The $55 \mu \mathrm{g}$ of sucrose per $1 \mathrm{~g}$ of $\mathrm{FW}$ difference, or $29 \%$ reduction, represents the portion of sucrose transported by basal endocytosis and accumulated in the vacuole. Figure 6 also demonstrates that at 1 $\mathrm{mm}$, the sucrose taken up into the cell by plasmalemma-bound carriers is not used exclusively for cytosolic metabolism but can also accumulate within the cell. Whether accumulation of the remaining sucrose takes place in the cytosol, the vacuole, or both, directly implicating the involvement of one or both membrane-bound sucrose transporters cannot be determined with the present observations.

The data of Fig. 6 also presents a convincing argument against the possibility that the inhibitory effect of the actin-depolymerizing agent latrunculin-B on sucrose uptake could be attributed to the prevention of plasmalemma-bound, sucrose-carrier recycling. The fact that sucrose uptake remained linear after $5 \mathrm{~h}$ in the presence of latrunculin$\mathrm{B}$ and $1 \mathrm{~mm}$ sucrose (where carrier-mediated transport of sucrose dominates) indicates that carrier-mediated sucrose transport was unaffected by the paralysis of membrane recycling. 


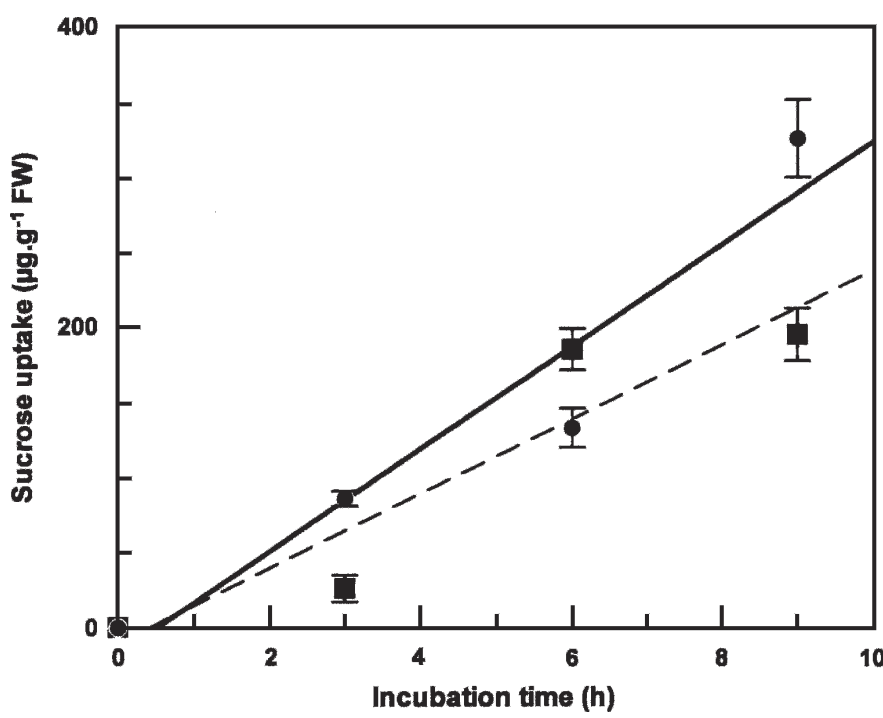

Fig. 6. Sucrose accumulation in turnip storage parenchyma cells incubated in 1 $\mathrm{mm}$ sucrose and the absence $(\bullet)$ and presence of $10 \mu \mathrm{M}$ latrunculin-B (ם). Tissue samples were preincubated for $30 \mathrm{~min}$ with and without $10 \mu \mathrm{M}$ latrunculin-B before the addition of sucrose. Due to the low external sucrose concentration, incubation was carried out for $10 \mathrm{~h}$. Data points were linearized based on (A) linear uptake of sucrose vs. the time used to generate Fig. 1, and (B) the premise that endocytosis transport results in linear uptake with time and concentration (Aniento and Robinson, 2005; Holstein, 2002).

\section{Discussion}

Sugar uptake into plant storage cells is accomplished by the complex interaction of several mechanisms, including plasmalemma- (Lalonde et al., 2004) and tonoplast-bound carriers (Endler et al., 2006; Manning et al., 2001; Shakya and Sturm, 1998), sugar facilitators (Overvoorde et al., 1996), and FPE (Baluska et al., 2006). Together with evidence presented in a series of papers (Baroja-Fernandez et al., 2006; Etxeberria et al., 2005a, 2005b, 2006), results presented in this article are the strongest indication that two metabolically dependent uptake systems operate to transport sucrose into storage cells. The data strengthen the significant role FPE plays in sucrose uptake (Baroja-Fernandez et al., 2006; Etxeberria et al., 2005a, 2005b, 2006), more specifically at concentrations characterized by the nonsaturable linear phase. When the overall data of sucrose and Alexa-488 uptake and accumulation are analyzed jointly (Figs. 1-3), an unmistakable view of the involvement of FPE in the two transport phases becomes apparent. The strong inhibitory effect exerted by phloridzin within the hyperbolic phase of the concentration curve (Fig. 2) and the data of Fig. 6, strongly indicate that under conditions of low external sucrose concentration, most sucrose accumulating in the cell enters via plasmalemma-bound carrier(s) and only a small contribution is sustained by endocytic uptake. By contrast, the increasing inhibitory effect exerted by latrunculin-B within the linear phase of the concentration curve strongly indicates that above a certain threshold external sucrose concentration, plasmalemma-bound carriers and FPE are determinants of sucrose accumulation in the cell. FPE appears to be a gradually superimposed transport system over carrier-mediated transport, its overall involvement increasing as the external sucrose concentration increases. The transfer of external solutes to the vacuole in parallel with the intense tonoplast fluorescence originating at the plasmalemma observed in Figs. 4 and 5 verifies the endocytic uptake of sucrose at the higher external concentration predicted by Fig. 3. It must be pointed out that the data presented here do not rule out a limited participation by passive diffusion to the overall process of sucrose accumulation at higher external sucrose concentrations, although in other systems such as yeast (Saccharomyces cerevisiae Meyen ex E.C. Hansen), it has been recognized that that low-affinity linear component of glucose transport is not due to passive diffusion (Gamo et al., 1995).

The relative contribution of FPE to the overall process of sucrose transport into the vacuole at low external concentrations is likely underestimated by the data of Figs. 1-3. The deceptive underestimate results from the fact that our data are based on accumulation rather than transport rates. Whereas accumulation of sucrose in the cytosol is determined by an equilibrium between uptake, synthesis and degradation (Geigenberger and Stitt, 1991, 1993; Wendler et al., 1990), vacuolar accumulation of sucrose must occur only when uptake rates surpass unidirectional enzymatic hydrolysis. These distinctive metabolic conditions would create an apparent delay in sucrose accumulation in the vacuole relative to its transport and would result in an artifactual shift toward the higher end of the linear phase (Fig. 1). The magnitude of this shift depends on the balance between the rate of vesicular transport of sucrose to the vacuole and the in vivo rates of vacuolar sucrose hydrolysis.

The dynamic involvement amongst transport systems is further complicated by other factors such as species-specific saturation kinetics, developmental stage, and osmolarity. For example, in sycamore cells, wortmannin-A reduced sucrose accumulation $\approx 90 \%$ at $50 \mathrm{~mm}$ external sucrose (Etxeberria et al., 2005a), strongly suggesting that the high-affinity component in turnip hypocotyl cells saturates at much higher concentration than in sycamore. In addition, rates of accumulation at $1 \mathrm{~mm}$ external sucrose and $1 \mathrm{~mm}$ endocytic marker were very similar during prolonged culture conditions (Etxeberria et al., 2005a), strongly indicating that even under conditions of low external sucrose concentration, FPE strongly determines the intracellular sucrose levels in sycamore cells.

The type of curve (linear/hyperbolic) also depends on the molarity of incubating (apoplastic) solution (Zamski, 1996). The hyperbolic phase of the sucrose accumulation curve is inhibited by increased cell turgor (Oliveira et al., 2002; Wyse et al., 1986; Zamski, 1996), making the linear component more relevant at higher external concentrations, an observation consistent with our results. However, incubation of sugar beet (Beta vulgaris L.) leaves in $100 \mathrm{~mm}$ sucrose reduced sucrose transporter transcripts and decreased transport activity by $35 \%$ to $50 \%$ (Chiou and Bush, 1998).

Finally, transport kinetics varies throughout development. In developing Vicia faba L. cotyledons for instance, the hexose uptake curve fluctuates in magnitude as the tissue matures, with the contribution by the hexose/symporter saturation phase declining as the tissue matures from a growth phase to accumulation phase (Harrington et al., 2005). It is likely that in a resting storage organ such as the mature turnip hypocotyl, the membrane-bound carriers are a higher component of the transport mechanism than in actively accumulating tissues such as sycamore cultured cells, where endocytosis predominates. Therefore, the extent by which FPE contributes to the overall uptake of photoassimilates into storage cells is a dynamic process throughout development that becomes more prominent as the concentration of sucrose increases in the apoplastic space. 


\section{Literature Cited}

Aniento, F. and D. Robinson. 2005. Testing for endocytosis in plants. Protoplasma 226:3-11.

Baluska, F., E. Baroja-Fernandez, J. Pozueta-Romero, A. Hlavacka, E. Etxeberria, and J. Samaj. 2006. Endocytic uptake of nutrients, cell wall molecules and fluidized cell wall potions in heterotrophic plant cells, p. 19-35. In: J. Smaj, F. Baluska, and D. Menzel (eds.). Plant endocytosis. Springer-Verlag, Berlin-Heidelberg, Germany.

Baluska, F., A. Hlavacka, J. Smaj, K. Palme, D.R. Robinson, T. Matoh, D.W. McCurdy, D. Menzel, and D. Volkmann. 2002. F-actindependent endocytosis of cell wall pectins in meristematic root cells. Insights from brefeldin A-induced compartments. Plant Physiol. 130:422-431.

Baluska, F., J. Samaj, A. Hlavacka, J. Kendrick-Jones, and D. Volkmann. 2004. Actin-dependent fluid-phase endocytosis in inner cortex cells of maize root apices. J. Expt. Bot. 55:463-473.

Baroja-Fernandez, E., E. Etxeberria, J.F. Muñoz, P. Gonzalez, and J. Pozueta-Romero. 2006. An important pool of sucrose linked to starch biosynthesis is taken up by endocytosis in heterotrophic cells. Plant Cell Physiol. 47:447-456.

Basrai, M.A., F. Naider, and J.M. Becker. 1990. Internalization of Lucifer yellow in Candida albicans by fluid phase endocytosis. J. Gen. Microbiol. 136:1059-1065.

Bieleski, R.L. 1960. The physiology of sugarcane. III. Characteristics of sugar uptake in slices of mature and immature storage tissue. Aust. J. Biol. Sci. 13:203-220.

Bush, D. 1993. Inhibitors of the proton-sucrose symport. Arch. Biochem. Biophys. 307:355-360.

Chapleo, S. and J.L. Hall. 1989. Sugar unloading in roots of Ricinus communis L. II. Characteristics of extravascular apoplast. New Phytol. 11:381-390.

Chiou, T.J. and D.R. Bush. 1998. Sucrose is a signal molecule in assimilate partitioning. Proc. Natl. Acad. Sci. USA 95:4784-4788.

Daie, J. 1984. Characterization of sugar transport in storage tissue of carrot. J. Amer. Soc. Hort. Sci. 109:718-722.

Daie, J. 1986. Kinetics of sugar transport in isolated vascular bundles and phloem tissue of celery. J. Amer. Soc. Hort. Sci. 111: 216-220.

Emans, N., S. Zimmermann, and R. Fischer. 2002. Uptake of a fluorescent marker in plant cells sensitive to brefeldin-A and wortmannin. Plant Cell 14:71-86.

Endler, A., S. Meyer, S. Scelbert, T. Scheider, W. Weschke, S.W. Peters, F. Keller, S. Baginsky, E. Martinoia, and U.G. Schmidt. 2006. Identification of a vacuolar sucrose transporter in barley and Arabidopsis mesophyll cells by tonoplast proteomic approach. Plant Physiol. 141:196-207.

Etxeberria, E., E. Baroja-Fernandez, F.J. Muñoz, and J. PozuetaRomero. 2005a. Sucrose inducible endocytosis as a mechanism for nutrient uptake in heterotrophic plant cells. Plant Cell Physiol. 46:474-481.

Etxeberria, E., P. Gonzalez, E. Baroja-Fernandez, and J. PozuetaRomero. 2006. Fluid phase uptake of artificial nanospheres and fluorescent quantum-dots by sycamore cultured cells. Plant Signaling Behaviour 1:181-185.

Etxeberria, E., P. Gonzalez, and J. Pozueta-Romero. 2005b. Sucrose transport into the vacuole of Citrus juice cells: Evidence for an endocytic transport system. J. Amer. Soc. Hort. Sci. 130:269-274.

Etxeberria, E., P. Gonzalez, and J. Pozueta-Romero. 2007a. Mannitol enhanced fluid-phase endocytosis in storage parenchyma cells of celery (Apium graveolens) petioles. Amer. J. Bot. 94:1043-1047.

Etxeberria, E., P. Gonzalez, and J. Pozueta-Romero. 2007b. Fluidphase endocytosis in citrus juice cells is independent from vacuolar $\mathrm{pH}$ and inhibited by chlorpromazine, an inhibitor of PI-3 kinases and clathrin-mediated endocytosis. J. Hort. Sci. Biotechnol. 82:900-907. Etxeberria, E., P. Gonzalez, P. Tomlinson, and J. Pozueta-Romero. 2005c. Existence of two parallel mechanisms for glucose uptake in heterotrophic plant cells. J. Expt. Bot. 56:1905-1912.
Fernie, A.R., J.W. Riesmeier, J.W. Martini, S. Ramalingan, L. Willmitzer, and R.N. Trethewey. 2000. Consequences of the expression of a bacterial glukokinase in potato tubers, both in combination with and independently of a yeast derived invertases. Aust. J. Plant Physiol. 27:827-833.

Fischer-Parton, S., R.M. Parton, P.C. Hickey, J. Dijksterhuis, H.A. Atkinson, and N.D. Read. 2000. Confocal microscopy of FM 4-64 as a tool for analyzing endocytosis and vesicle trafficking in living fungal hyphae. J. Microsc. 198:246-259.

Gamo, F.J., E. Moreno, and R. Lagunas. 1995. The low-affinity component of the glucose transport system in Saccharomyces cerevisiae is not due to passive diffusion. Yeast 11:1393-1398.

Geigenberger, P. and M. Stitt. 1991. A "futile" cycle of sucrose synthesis and degradation is involved in regulating partitioning between sucrose, starch and respiration in cotyledons of germinating Ricinus communis L. seedlings when phloem transport is inhibited. Planta 185:81-90.

Geigenberger, P. and M. Stitt. 1993. Sucrose synthase catalyses a readily reversible reaction in vivo in developing potato tubers and other plant tissues. Planta 189:329-339.

Getz, H.P. and M. Klein. 1995. Characteristics of sucrose transport and sucrose-induced $\mathrm{H}^{+}$transport on the tonoplast of red beet (Beta vulgaris L.) storage tissue. Plant Physiol. 107:459-467.

Harrington, G.N., K.E. Dibley, R. Ritchie, C.E. Offler, and J.W. Patrick. 2005. Hexose uptake by developing cotyledons of Vicia faba: Physiological evidence for transporters of different affinities and specificities. Funct. Plant Biol. 32:987-995.

Haug, A. and B. Shi. 1991. Biochemical basis of aluminum tolerance in plant cells. Dev. Plant Soil Sci. 45:839-845.

Holstein, S. 2002. Clathrin and plant endocytosis. Traffic 3:614-620. Lalonde, S., D. Wipf, and W. Frommer. 2004. Transport mechanisms for organic forms of carbon and nitrogen between source and sink. Annu. Rev. Plant Biol. 55:341-372.

Lalonde, S.L., E. Boles, H. Hellmann, L. Barker, J.W. Patrick, W.B. Frommer, and J.M. Ward. 1999. The dual function of sugar carriers: Transport and sugar sensing. Plant Cell 11:707-726.

MacRobbie, E.A.C. 1999. Vesicle trafficking: A role in trans-tonoplast ion movements? J. Expt. Bot. 50:925-934.

Manning, K. 1998. Isolation of a set of ripening-related genes from strawberry: Their identification and possible relationship to fruit quality traits. Planta 205:622-631.

Manning, K., C. Davies, H.C. Bowen, and P.J. White. 2001. Functional characterization of two ripening-related sucrose transporters from grape berries. Ann. Bot. (Lond.) 87:125-129.

Niemietz, C. and C.F. Jenner. 1993. Mechanisms of sugar uptake into endosperm and aleurone protoplasts from developing wheat grains. Aust. J. Plant Physiol. 20:371-378.

Nishikawa, F., M. Kato, H. Hyodo, Y. Ikoma, M. Sugiura, and M. Yano. 2005. Effect of sucrose on ascorbate level and expression of genes involved in the ascorbate biosynthesis and recycling pathway in harvested broccoli florets. J. Expt. Bot. 56:65-72.

Oliveira, J., R.M. Tavares, and H. Geros. 2002. Utilization and transport of glucose in Olea europea cell suspensions. Plant Cell Physiol. 43:1510-1517.

Overvoorde, P.J., W. Frommer, and H.D. Grimes. 1996. A soybean sucrose binding protein independently mediates nonsaturable sucrose uptake in yeast. Plant Cell 8:271-280.

Piddini, E. and J.-P. Vincent. 2003. Modulation of developmental signals by endocytosis: Different means and many ends. Curr. Opin. Cell Biol. 15:474-481.

Robinson, D.R., G. Hinz, and S.H.E. Holstein. 1998. The molecular characterization of transport vesicles. Plant Mol. Biol. 38:4976.

Saftner, R.A. and J. Daie. 1983. Sucrose uptake and compartmentation in sugar beet taproot tissue. Plant Physiol. 72:1-6.

Shakya, R. and A. Sturm. 1998. Characterization of sucrose- and sinkspecific sucrose $/ \mathrm{H}^{+}$symporters from carrot. Plant Physiol. 118:14731480 . 
Shimoaka, T., M. Ohnishi, T. Sazuka, N. Mitsuhashi, I. Hara-Nishimura, K.-I. Shimazaki, M. Maeshima, A. Yokota, K.-I. Tomizawa, and T. Mimura. 2004. Isolation of intact vacuoles and proteomic analysis of tonoplast from suspension-cultured cells of Arabidopsis thaliana. Plant Cell Physiol. 45:672-683.

Sorkin, A. and M. von Zastrow. 2002. Signal transduction and endocytosis: Close encounters of many kinds. Nat. Rev. Mol. Cell Biol. 3:600-614.

Van Handel, E. 1968. Direct microdetermination of sucrose. Ann. Biochem. 22:280-283.

Wendler, R., R. Veith, J. Dancer, M. Stitt, and E. Komor. 1990. Sucrose storage in cell suspension cultures of Saccharum sp. (sugarcane) is regulated by a cycle of synthesis and degradation. Planta 183:31-39.

Wright, K. and K.J. Oparka. 1989. Sucrose uptake and partitioning in discs derived from source versus sink potato tubers. Planta 177:237-244.

Wyse, R. 1979. Sucrose uptake by sugar beet tap root tissue. Plant Physiol. 64:837-841.

Wyse, R., E. Zamski, and A.D. Tomos. 1986. Turgor regulation of sucrose transport in sugar beet taproot tissue. Plant Physiol. 81:478-481.

Zamski, E. 1996. Anatomical and physiological characteristics of sink cells, p. 283-310. In: E. Zamski and A.A. Schaffer (eds.). Photoassimilate distribution in plants and crops. Source-sink relationships. Marcel Dekker, New York. 